\title{
Dynamics of Quantum Phase Transition in an Array of Josephson Junctions
}

\author{
J. Dziarmaga ${ }^{1,2}$, A. Smerzi ${ }^{1,3}$, W.H. Zurek ${ }^{1}$, and A.R. Bishop ${ }^{1}$ \\ 1) Los Alamos National Laboratory, Theory Division, Los Alamos, NM 87545, USA \\ 2) Instytut Fizyki Uniwersytetu Jagiellońskiego, Reymonta 4, 30-059 Kraków, Poland \\ 3) Istituto Nazionale di Fisica per la Materia and International School for Advanced Studies, \\ via Beirut 2/4, I-34014, Trieste, Italy
}

(October 30, 2001)

We study the dynamics of the Mott insulator-superfluid quantum phase transition in a periodic 1D array of Josephson junctions. We show that crossing the critical point at a finite rate with a quench time $\tau_{Q}$ induces finite quantum fluctuations of the current around the loop proportional to $\tau_{Q}^{-1 / 6}$. This scaling could be experimentally verified with in array of weakly coupled Bose-Einstein condensates or superconducting grains.

74.50.+r,11.30.Qc,03.75.Fi

Thermal fluctuations critically slow down close to a continuous symmetry breaking thermodynamic phase transition. Both the relaxation time $\tau$ and the correlation length $\xi$ diverge when a dimensionless parameter $\epsilon$, which measures the distance from the critical point, tends to zero: $\xi \sim|\epsilon|^{-\nu}$ and $\tau \sim|\epsilon|^{-z \nu}$, with $z$ and $\nu$ critical exponents. In the infinitesimally slow transition the symmetry broken phase is entered in a state of equilibrium with a fully ordered complex order parameter. However, in a quench occuring at a finite rate transition the critical slowing down $(\tau \rightarrow \infty$ when $\epsilon \rightarrow 0)$ implies that the system goes out of equilibrium some time before the transition. As a result the complex order parameter which emerges after the transition will assume different random phases in different domains of space. This leads to formation of topological defects such as vortices wherever the circulation of the phase around a closed loop happens to be nonzero. This process is known as Kibble-Zurek mechanism (KZM) 股化. The size of the domains follows from a simple argument [3]: Close to the transition one can linearize the time dependence of $\epsilon$ as $\epsilon(t) \approx t / \tau_{Q}$. The transition rate is $r(t)=\dot{\epsilon} / \epsilon=1 /|t|$. The system goes out of equilibrium at the time $-\hat{t}$ before the transition when $r(-\hat{t})=\tau^{-1}(-\hat{t})$. After that time the state of the system essentially does not change until $+\hat{t}$, when the rate $r(t)$ becomes again equal to the relaxation rate, $r(+\hat{t})=\tau^{-1}(+\hat{t})$. At $\hat{t}=\tau_{Q}^{z \nu /(1+z \nu)}$ fluctuations of the order parameter with wavelengths longer than $\hat{\xi}=\xi(\hat{t})=\tau_{Q}^{\nu /(1+z \nu)}$ begin to grow exponentially while short wavelength fluctuations remain unchanged. The size of the correlated domains is given by $\hat{\xi}$ and the density of vortices by $1 / \hat{\xi}^{2}$.

Theoretical and experimental studies of KZM have concentrated so far on thermal continuous phase transitions with the dynamics of the order parameter governed by an effective irreversible time-dependent GinzurgLandau theory. Most attention has been devoted to the normal-superfluid transition in ${ }^{3} \mathrm{He}$ [6], superconductors [5], and, more recently, dilute Bose-Einstein condensates [7]. To date, truly microscopic quantum approaches have been too complicated to extract useful predictions.

A quench-induced quantum phase transition (QPT) at temperature $T=0$ must be treated in a microscopic way. It is a common wisdom that some properties of a quantum transition can be obtained by an exact map from a thermodynamic transition [1]: the correlation length in the ground state of the quantum system scales like $\xi \sim|\epsilon|^{-\nu}$ and the gap between the ground state and the first excited state like $d E \sim|\epsilon|^{-z \nu}$. To study the dynamics of a quantum transition, which drives the system out of its ground state, we also need information about correlations in excited states, which is not provided by this map. As we will see below, the essence of the KZM, which is the competition between the transition rate and the timescale on which the system can react, is applicable to quantum transitions. However, the quantum scenario and in particular interpretation of its results differ from the thermal case. The main reason is the reversibility of the quantum dynamics, as opposed to the dynamical irreversibility of the thermal critical dynamics.

In this Letter, we study the appearance of a nonzero current while the system is undergoing a (zerotemperature) quantum phase transition. We develop a microscopic, dynamical theory and suggest experiments to test our predictions.

Josephson junction arrays. A prototype system displaying a continuous QPT is an array of mesoscopic Josephson junctions (JJ). Superconducting JJ networks are now available thanks to important advances in techniques for ultra-small superconducting grains although existence of gauge fields may complicate the analysis 国. On the other hand, manipulation of optically trapped Bose-Einstein condensates promises the observation of QPT in neutral quantum fluids [10,11]. In such systems, a superconducting/superfluid-insulator phase transition is driven by the competition between two physical magnitudes: the Josephson coupling energy $E_{J}$, which governs the tunneling through the intra-well barriers, and the onsite interparticle interaction energy $E_{C}$. When they are 
comparable, there is a competition between long-range order (which is favoured by $E_{J}$ ) and localization (induced by $E_{C}$ ). When the latter prevails, no net current can flow through the junctions.

An effective Hamiltonian describing the quantum dynamics of an array of JJ's is given by the Quantum Phase Model (QPM) [12]:

$$
\hat{H}=\sum_{l=1}^{N_{s}} \frac{E_{C}}{2} \hat{n}_{l}^{2}-\sum_{\langle k, l\rangle} E_{J} \cos \left(\hat{\phi}_{k}-\hat{\phi}_{l}\right)
$$

with the last sum running over the nearest-neighbour sites. For our purposes, it is enough to consider the case of a diagonal charging energy matrix $E_{c}$, independent of the site indices. The phase $\hat{\phi}_{l}$ and the number of atoms $\hat{n}_{l}$ in each site of the junction are (with some important caveats 13) non-commuting conjugate observables $\left[\hat{n}_{l}, \hat{\phi}_{l}\right]=i$, and, in the $\phi$-representation, $\hat{n}_{l} \equiv n-i \frac{\partial}{\partial \phi_{l}} ; \hat{\phi}_{l} \equiv \phi_{l}$. Therefore, $\phi_{l}$ and $n_{l}$ play the role of coordinate and conjugate momentum, and satisfy the Heisenberg uncertainty relation The insulator phase is characterized by large quantum phase fluctuations in the ground state, which destroy the long-range order among sites. The 1D Hamiltonian Eq.(1) exhibits a continuous Mott phase transition at the critical value $G_{c} \equiv E_{J} / E_{C}=0.617$ [1]. In what follows we study the microscopic dynamics of quantum phase transitions in a one-dimensional chain with periodic boundary conditions.

The dynamics is governed by the QPM Hamiltonian Eq.(11), and satisfies a Schrödinger equation

$$
i \frac{\partial}{\partial t} \Psi=-\frac{1}{2} \sum_{l=1}^{N_{s}} \frac{\partial^{2}}{\partial \phi_{l}^{2}} \Psi-G \sum_{\langle k, l\rangle} \cos \left(\phi_{k}-\phi_{l}\right) \Psi
$$

with $G=E_{J} / E_{c}$ and the time rescaled as $t \rightarrow E_{c} t$. This equation is valid for a large number of atoms per site, $n \gg 1$, and for $G \ll n^{2}[14$. The ground state below the transition $\left(G<G_{c}\right)$ is close to the Fock state $|n, n, n, \ldots\rangle$ with all sites occupied by the same number of atoms $n$. In the phase representation this state is described by a uniform wavefunction, $\Psi\left(\phi_{l}\right)=$ const, where all phase differences between nearest neighbor sites have the maximal dispersion of $\Delta \phi \sim 1$. Above the transition $\left(G>G_{c}\right)$ the ground state is a number squeezed state which continuously tends to a Fock state when $G \rightarrow G_{c}^{+}$, and to a coherent state for $G \gg n^{2}$. Therefore, when $G \gg G_{c}$ one can describe the low energy part of the spectrum of Eq.(2) in a harmonic approximation [15]

$$
i \frac{\partial}{\partial t} \Psi=-\frac{1}{2} \sum_{l=1}^{N_{s}} \frac{\partial^{2}}{\partial \phi_{l}^{2}} \Psi+\frac{G}{2} \sum_{\langle k, l\rangle}\left(\phi_{k}-\phi_{l}\right)^{2} \Psi .
$$

This equation is diagonalized by normal modes numbered by momentum $\mu \in\left\{-N_{s}+1, \ldots,+N_{s}\right\}, \Psi=$ $\prod_{\mu} \Psi_{\mu}\left(\Phi_{\mu}\right)$. There is one zero mode $\Phi_{0} \sim \sum_{l} \phi_{l}$. All other modes $\Phi_{\mu}$ have nonzero frequencies $\sqrt{\gamma_{\mu} G}[\approx$ $G^{1 / 2}\left(\frac{2 \pi|\mu|}{N_{s}}\right)$ for small $\left.\mu\right]$, which scale as $G^{1 / 2}$,

$$
i \frac{\partial}{\partial t} \Psi_{\mu}=-\frac{1}{2} \frac{\partial^{2}}{\partial \Phi_{\mu}^{2}} \Psi_{\mu}+\frac{\gamma_{\mu} G}{2} \Phi_{\mu}^{2} \Psi_{\mu}
$$

To implement quench with a quench timescale $\tau_{Q}$ we linearly ramp the control parameter $G$ in Eq.(2) as

$$
G(t)=\frac{t}{\tau_{Q}}
$$

The time $t$ runs from 0 to $\tau_{Q} G_{\max }$ with $G_{\max } \gg G_{c}$.

Adiabatic transition. In the limit $\tau_{Q} \rightarrow \infty$ the transition is adiabatic and the system state adiabatically follows its ground state from the Fock state $|n, \ldots, n\rangle$ at $G=0$ to a coherent state with all the nearest neighbor phase differences close to zero, $\Delta \phi \approx 0$, for $G_{\max } \gg G_{c}$. The ground state at $G_{\max } \gg G_{c}$ is well described by the ground state of the harmonic oscillators (-4). The dispersion of phases in any harmonic oscillator ground state at $G_{\max }$ is proportional to $G_{\max }^{-1 / 4}$. The final dispersion of phase differences in the adiabatic transition is

$$
\Delta \phi_{\tau_{Q} \rightarrow \infty} \simeq G_{\max }^{-1 / 4}
$$

Instantaneous transition. In the opposite limit of an istantaneous transition, when $\tau_{Q} \rightarrow 0$, the system is not able to adjust its quantum state to the changing hopping rate $G(t)$, and it remains in the initial Fock state till $G_{\max }$. The final dispersion of the phase differences in the uniform Fock state, $\Psi\left(\phi_{l}\right)=$ const, is

$$
\Delta \phi_{\tau_{Q} \rightarrow 0} \sim 1
$$

For a generic quench the evolution of the system is approximately adiabatic when $G$ is far from $G_{c}$, and impulse when $G$ is close to $G_{c}$; compare Fig.1 for a 3-site periodic lattice. This is similar to the thermal case [3]. As $G$ increases starting from 0 the system initially follows its ground state, which is an incoherent superposition of Fock states, until a $\hat{G}_{-}<G_{c}$ when the transition ceases to be adiabatic. Between $\hat{G}_{-}$and a certain $\hat{G}>G_{c}$ the evolution is impulse: to a first approximation the state of the system does not change. The system arrives at $\hat{G}$ in an incoherent superposition of Fock states with a dispersion $\Delta \phi \sim 1$ as in the initial Fock state. After $\hat{G}$ the transition again becomes adiabatic.

Fast transitions with $\left(E_{c}\right) \tau_{Q} \ll 1$. Let us estimate $\hat{G}$ for a dimensionless $\tau_{Q} \ll 1$ when we expect that $\hat{G} \gg G_{c}$ and we can use the harmonic approximation (3, 4) for $G \approx \hat{G}$. For the linear quench (5) the periods of the oscillators decay like $G(t)^{-1 / 2}=\sqrt{\tau_{Q} / t G_{\max }}$. The transition timescale is $G / \dot{G}=t$. This rate becomes comparable to the periods of the oscillators at the time $\hat{t}$ 
when $G^{-1 / 2} \simeq G / \dot{G}$ : compare Fig.1 for a 3-site periodic lattice. A solution of this equation gives

$$
\begin{aligned}
& \hat{t}_{\tau_{Q} \ll 1} \simeq \tau_{Q}^{1 / 3}\left(\text { or } E_{c}^{-2 / 3} \tau_{Q}^{1 / 3}\right), \\
& \hat{G}_{\tau_{Q} \ll 1} \equiv G\left(\hat{t}_{\tau_{Q} \ll 1}\right) \simeq \tau_{Q}^{-2 / 3} .
\end{aligned}
$$

As anticipated, when $\tau_{Q} \ll 1$ we have $\hat{G}_{\tau_{Q} \ll 1} \gg G_{c}$.

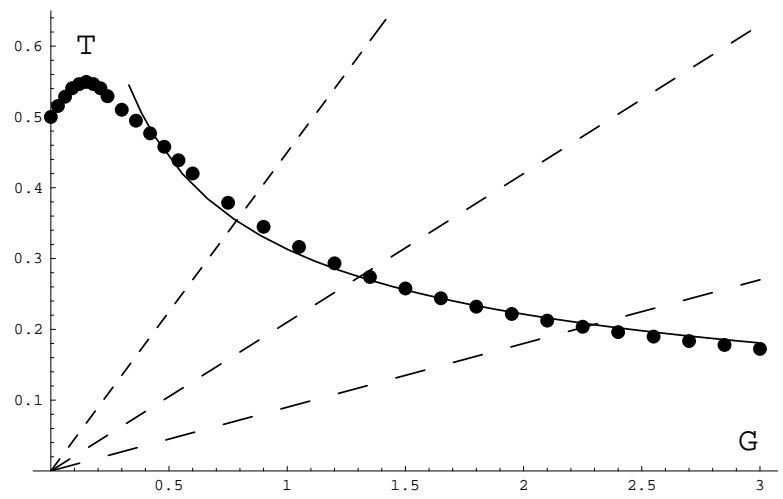

FIG. 1. The inverse of the gap $T=1 / d E$ as a function of $G$ for a 3 -site periodic Hubbard model with $n=6$ atoms per site (dots). The solid line is the best $G^{-1 / 2}$ fit. The dashed lines show the transition time $G / \dot{G}=t=G \tau_{Q} / G_{\max }$ for $G_{\max }=10$ and $\tau_{Q}=4.5,2.1,0.9$ (from left to right). The crossings between $1 / d E$ and the dashed lines define $\hat{G}$ for the different $\tau_{Q}$.

We can use the fact that $\hat{G}_{\tau_{Q} \ll 1}$ to estimate the final dispersion $\Delta \phi$ at $G_{\max }$. At $\hat{G}_{\tau_{Q} \ll 1}$ the dispersion is $\Delta \phi \sim 1$. For $G>\hat{G}_{\tau_{Q} \ll 1}$ the evolution of the harmonic oscillators is adiabatic. There is no mixing between the eigenstates of any oscillator $\mu$ (1). The dispersion of the phase $\Phi_{\mu}$ in any given eigenstate of the oscillator (14) scales as $G^{-1 / 4}$. In the adiabatic evolution for $G(t)>\hat{G}_{\tau_{Q} \ll 1}$ the dispersion of $\Phi_{\mu}$ shrinks like $\left[\hat{G}_{\tau_{Q} \ll 1} / G(t)\right]^{1 / 4} . \Delta \phi$ shrinks in the same way. The final dispersion at $G_{\max }$ is $\Delta \phi \simeq\left[\hat{G}_{\tau_{Q} \ll 1} / G_{\max }\right]^{1 / 4}$ or

$$
\Delta \phi_{\tau_{Q} \ll 1} \simeq \tau_{Q}^{-1 / 6} G_{\max }^{-1 / 4}\left(\text { or } E_{c}^{-1 / 6} \tau_{Q}^{-1 / 6} G_{\max }^{-1 / 4}\right) .
$$

In a $1 \mathrm{D}$ periodic lattice this $\Delta \phi_{\tau_{Q} \ll 1}$ translates into a dispersion of the angular momentum $L=i \sum_{l}\left(a_{l}^{\dagger} a_{l+1}-\right.$ $a_{l+1}^{\dagger} a_{l}$ ) through the formula

$$
\Delta L \sim \sqrt{N_{s}} n \Delta \phi
$$

This $\Delta L$ may translate into dispersion of the winding number $\sqrt{N_{s}} \Delta \phi$ when the atoms after the quench are forced to condense. Eq. (10) is valid when the predicted $\hat{G}_{\tau_{Q} \ll 1} \simeq \tau_{Q}^{-2 / 3}$ is much less than the final $G_{\max }$. This condition is satisfied when $\tau_{Q} \gg G_{\max }^{-2 / 3}$. In fact for the slowest $\mu= \pm 1$ modes to become adiabatic before the maximal possible $G_{\max }=n^{2}$ we need $\tau_{Q} \gg n / N_{s}$.

Figures 2 and 3 show results of numerical simulations of the 3 -site periodic lattice with a total of 300 atoms.
In Fig. 2 we show that $\Delta \phi$ remains the same as in the initial Fock state for $G<\hat{G}_{\tau_{Q} \ll 1}$. In Figure 3 we verify the scaling $\hat{t} \sim \tau_{Q}^{1 / 3}$. We also performed similar simulations for a total of 120 (commensurate) and $120+1$ atoms (non-commensurate density of atoms). Plots like those in Fig.2,3 for 120 and 121 atoms are impossible to distinguish which shows that an extra noncommensurate atom does not make any difference for fast transitions.

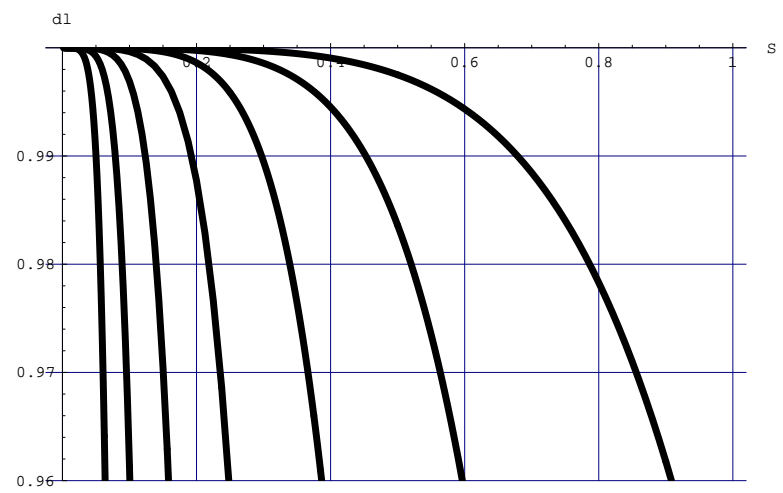

FIG. 2. The normalized dispersion $d l=\Delta L / \Delta L_{\tau_{Q} \rightarrow 0}$ as a function of $S=G(t) / G_{\max }$. We show results of exact numerical simulations of the 3-site Hubbard model for 7 different $\tau_{Q}=0.016,0.008,0.004,0.002,0.001,0.0005,0.00025$ (from left to right) with $G_{\max }=1000$ and $n=100$ atoms per site. $d l$ deviates from 1 (and hits the bottom of the figure) at $\hat{G} \sim \tau_{Q}^{-2 / 3}$ : compare Fig.2.

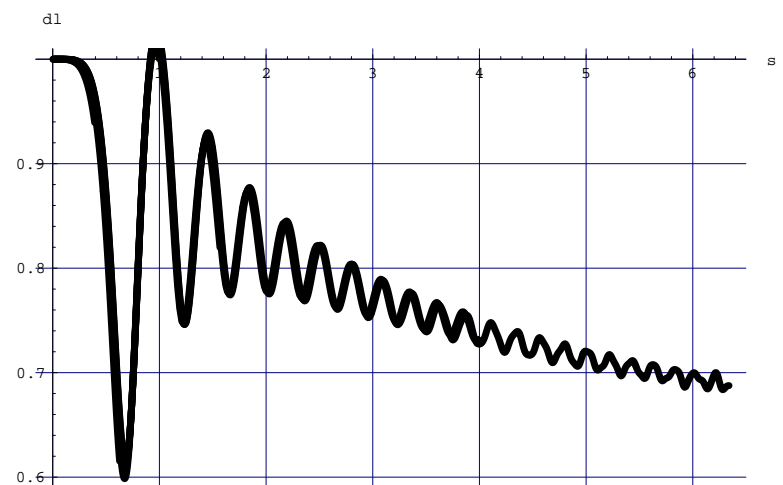

FIG. 3. The normalized dispersion $d l=\Delta L / \Delta L_{\tau_{Q} \rightarrow 0}$ as a function of $s=G(t) / \hat{G}$ with $\hat{G} \sim \tau_{Q}^{-2 / 3}$. This figure displays all the seven plots from Fig.2. The plots in Fig.2 are in the top left corner of this figure. The fact that the seven plots sit on top of each other proves that the evolution of $\Delta L$ depends on time $t$ through the combination $t / \hat{t}$ with $\hat{t} \sim \tau_{Q}^{1 / 3}$.

Slow transitions with $\left(E_{c}\right) \tau_{Q} \gg 1$. So far we concentrated on $\tau_{Q} \ll 1$ with $\hat{G}$ well in the harmonic regime. In slower transitions $\hat{G}$ is close to $G_{c}$ where the harmonic approximation cannot be applied. The slow transitions $\left(\tau_{Q} \ll 1\right)$ are probing the critical behaviour close to $G_{c}$.

In the perioric 3 -site array there is no phase transition but, rather, a crossover: the gap $d E$ is minimal at the crossover point $G_{c} \approx 0.5$ but it does not vanish there, 
compare Fig.1. For $\tau_{Q} \gg 1$ the transition is adiabatic and the system follows its ground state. The phase difference dispersion at $G_{\max } \gg G_{c}$ is the dispersion in the ground state of the harmonic oscillators (6). A thermal crossover transition was studied in 19.

In a large $2 \mathrm{D}$ array with a commensurate density of atoms there is a phase transition and not a crossover [1,20]. The gap $d E$ vanishes at $G=G_{c}$. Close to the critical point, $G \approx G_{c}$, the energy gap scales as $d E \sim\left(G-G_{c}\right)^{\nu}$, where $\nu=2 / 3$ is an exact renormalization group critical exponent. Note that in the harmonic regime for $G \gg G_{c}$ we have $d E \sim G^{1 / 2}$ which is consistent with a mean-field value of $\nu=1 / 2$. With a linearized $G(t)-G_{c}=\frac{t}{\tau_{Q}}$ the gap $\Delta E \sim\left(G-G_{c}\right)^{2 / 3}=\left(t / \tau_{Q}\right)^{2 / 3}$ becomes equal to the quench rate $r(t)=\dot{G} /\left(G-G_{c}\right)=$ $1 / t$ at $\hat{G} \simeq G_{c}+1 / \tau_{Q}^{3 / 5}$. Before $\hat{G}$ the system is in an incoherent superposition of Fock states. After $\hat{G}$ the evolution is adiabatic, and with increasing $G$ the phase dispersion shrinks together with the phase widths of the system eigenstates. The dispersion measured at $G_{\max } \gg G_{c}$, where we can use the harmonic approximation, is

$$
\Delta \phi_{\tau_{Q} \gg 1}^{2 \mathrm{D}} \approx \frac{\hat{G}^{1 / 4}}{G_{\max }^{1 / 4}} \simeq \frac{\left(G_{c}+\frac{1}{\left(E_{c}^{3 / 5}\right) \tau_{Q}^{3 / 5}}\right)^{1 / 4}}{G_{\max }^{1 / 4}} .
$$

This formula is consistent with Eq.(6) because $G_{c}=$ $\mathcal{O}(1)$. Here we again use the fact that the phase dispersion of harmonic oscillator eigenstates shrinks like $G^{-1 / 4}$. The critical behaviour is realized in the $\left(E_{c}^{3 / 5}\right) \tau_{Q}^{3 / 5}$ term.

Concluding remarks. The state of the system after the transition does not have definite angular momentum or definite phase differences between lattice sites. That would be the case in the thermal KZM, where $\Delta \phi$ would describe the dispersion in an ensemble of different possible classical outcomes. In a quantum phase transition at zero temperature the state of the system after the transition is in a coherent superposition of states with different $\Delta \phi$. Either a measurement or decoherence 21] are needed to convert this coherent superposition into a mixtures of states, each with definite current.

Another difference with respect to the thermal KZM is due to the reversibility of the quantum dynamics. In the thermal case the characteristic lenghtscale $\hat{\xi}$ is frozen after the symmetry breaking transition is completed. $\hat{\xi}$ is a permanent record of the transition rate $\tau_{Q}$ : manipulations with $\epsilon$ do not change the winding number as long as the system remains in the symmetry broken phase with $\epsilon>0$. In contrast, even after the diabatic quantum transition is completed one can change $\Delta \phi_{\tau_{Q}}$. Adiabatic variations of $G$ away from $G_{\max }$ are accompanied by changes in $\Delta \phi, \Delta \phi=\Delta \phi_{\tau_{Q}}\left(G_{\max } / G\right)^{1 / 4}$.

In conclusion, we have predicted the phase dispersion after a diabatic insulator-superfluid quantum phase transition in an array of Josephson junctions. This theory is a quantum counterpart of the Kibble-Zurek mechanism for topological defect formation in classical thermal phase transitions. Our predictions can be tested experimentally in $1 \mathrm{D}$ or $2 \mathrm{D}$ superconducting JJ arrays. The possibility of tuning the Josephson coupling energy in a superconducting JJ has been demonstrated recently [16]. 1D (or 3D 222) JJ arrays realized with Bose-Einstein condensates can be, perhaps even more easily, accurately tailored 10,11, 17, 18. For the case of a 1D array our Eq.(10) predicts a dispersion of the phases $\Delta \phi \simeq G_{\max }^{-1 / 4} \tau_{Q}^{-1 / 6}$ that can be directly measured by the interference techniques which have been used in the experiments [10,11, 17.

We acknowledge useful discussions with James Anglin and Diego Dalvit. This work was partially supported by the DOE and the Cofinanziamento MURST.

[1] S. Sachdev, Quantum Phase Transitions, Cambridge University Press, (1999).

[2] T.W.B. Kibble, J. Phys. A9, 1387 (1976); T.W.B. Kible and A. Vilenkin, Phys. Rev. D52, 679 (1995).

[3] W.H. Zurek, Nature (London) 317, 505 (1985).

[4] W.H. Zurek, Phys. Rep. 276, 177 (1996); M. Hindmarsh and A. Rajantie, Phys.Rev.Lett.85, 4660 (2000); G.J. Stephens, L.M.A. Bettencourt, and W.H. Zurek, cond-mat/0108127.

[5] R. Carmi et al., Phys. Rev. Lett.84, 4966 (2000); E. Kavoussanaki et al., Phys. Rev. Lett.85, 3452 (2000).

[6] V.M.H. Ruutu et al, Nature 382, 334 (1996); Bauerle et.al., Nature 382, 332 (1996).

[7] J.R. Anglin and W.H. Zurek, Phys.Rev.Lett. 83, 1707 (1999).

[8] S.L. Sondhi, S.M. Girvin, J.P. Carini, D. Shahar, Rev of Mod. Phys. 69, 315 (1997)

[9] A. Barone and G. Paterno, Physics and Applications of the Josephson Effect, Wiley, New York, 1982.

[10] B.P. Anderson and M.A. Kasevich, Science 282, 1686 (1998).

[11] C. Orzel, A.K. Tuchman, M.L. Fenslau, M. Yasuda and M.A. Kasevich, Science 291, 2386 (2001).

[12] E. Simanek, Inhomogeneous Superconductors, Oxford University Press, 1994.

[13] J. Anglin, P. Drummond and A. Smerzi, Phys.Rev A64, 063605 (2001)

[14] With this assumption, satisfied in the experiment [11, we could neglect in Eq.(2) the $O\left(G / n^{2}\right)$ correction terms derived in 13 .

[15] J. Javanainen, Phys. Rev. A60, 4902 (1999).

[16] J.H. Schon, C. Kloc, H.Y. Hwang and B. Batlogg, Science 292, 252 (2001).

[17] F.S. Cataliotti et.al., Science 293, 843 (2001).

[18] A. Trombettoni and A. Smerzi, Phys. Rev. Lett. 862353 (2001)

[19] W.H. Zurek, L.M.A. Bettencourt, J. Dziarmaga, and N.D. Antunes, Acta.Phys.Polon.B 32, 2279 (2001); see also J. Dziarmaga, Phys.Rev.Lett.81, 5485 (1998). 
[20] M.P.A. Fisher, P.B. Weichman, G. Grinstein, and D.S. Fisher, Phys.Rev.B 40, 546 (1989).

[21] W.H. Zurek, Phys. Today 44, 36 (1991); quant$\mathrm{ph} / 010527$.

[22] M. Greiner et al., Nature 415, 39 (2002). 